\title{
Green Synthesis of Cerium Oxide Nanoparticles $\left(\mathrm{CeO}_{2} \mathrm{NPs}\right)$ and Their Antimicrobial Applications: A Review
}

This article was published in the following Dove Press journal: International Journal of Nanomedicine

\author{
Muhammad Nadeem ' \\ Ramsha Khan' \\ Komal Afridi' \\ Akhtar Nadhman (D) \\ Sana Ullah $\mathbb{D}^{2}$ \\ Sulaiman Faisal ${ }^{1}$ \\ Zia UI Mabood (D) \\ Christophe Hano $\mathbb{1}^{3}$ \\ Bilal Haider Abbasi (iD) ${ }^{2}$ \\ 'Institute of Integrative Biosciences, \\ CECOS University of IT and Emerging \\ Sciences, Peshawar 25I00, KPK, Pakistan; \\ ${ }^{2}$ Department of Biotechnology, Quaid \\ I Azam University, Islamabad 45320, \\ Pakistan; ${ }^{3}$ Laboratoire de Biologie des \\ Ligneux et des Grandes Cultures \\ (LBLGC), Plant Lignans Team, INRAE \\ USCI328, Université d'Orléans, Eure Et \\ Loir Campus, Chartres F28000, France
}

Correspondence: Muhammad Nadeem Email m.nadeem@cecos.edu.pk

\begin{abstract}
During the last decade green synthesized cerium oxide nanoparticles $\left(\mathrm{CeO}_{2} \mathrm{NPs}\right)$ attracted remarkable interest in various fields of science and technology. This review, explores the vast array of biological resources such as plants, microbes, and other biological products being used in synthesis of $\mathrm{CeO}_{2} \mathrm{NPs}$. It also discusses their biosynthetic mechanism, current understandings, and trends in the green synthesis of $\mathrm{CeO}_{2} \mathrm{NPs}$. Novel therapies based on green synthesized $\mathrm{CeO}_{2} \mathrm{NPs}$ are illustrated, in particular their antimicrobial potential along with attempts of their mechanistic elucidation. Overall, the main objective of this review is to provide a rational insight of the major accomplishments of $\mathrm{CeO}_{2} \mathrm{NPs}$ as novel therapeutics agents for a wide range of microbial pathogens and combating other diseases.
\end{abstract}

Keywords: nanotechnology, green synthesis, cerium oxide, nanoparticles, antimicrobial, infections, biomedical

\section{Introduction}

Nanotechnology has got a remarkable interest in every field of science and technology and is presently considered among one of the leading research avenues. It has a multitude of applications in the field of electronics, imaging, industry, and healthcare. ${ }^{1,4}$ Mostly, in healthcare it has been exploited in diseases diagnostics, treatment, delivery, and formulations of novel drugs. ${ }^{1,4}$ It exploits nano size structures with size ranges from 1-100 nm, known as nanoparticle (NPs). These nanoscale entities have unique physiochemical properties and have been utilized in various fields of physics, biology, and chemistry. ${ }^{5}$

Among other NPs, Cerium Oxide $\left(\mathrm{CeO}_{2}\right)$ NPs have been mostly exploited due to their unique surface chemistry, high stability, and biocompatibility. ${ }^{6,7}$ It is mostly used in the fabrication of sensors, cells, catalysis, therapeutics agents, drug delivery careers, and anti-parasitic ointments (Figure 1). ${ }^{1,6,8}$ Presently, $\mathrm{CeO}_{2} \mathrm{NPs}$ is mostly synthesized via two methods, such as physical and chemical. ${ }^{7,9}$ However, these methods utilize toxic reducing solvents posing several threats to the biodiversity and ecosystem. Moreover, the NPs obtained with such approaches are toxic and unstable, making them less efficient. ${ }^{9,10}$ Thus, recently a safe, less toxic method has been used by researchers known as Green Synthesis. This method utilizes various biological resources such as plants, microbes, or any other biological derivative. $^{11,15}$ These biological extracts have a rich source of phytochemicals 


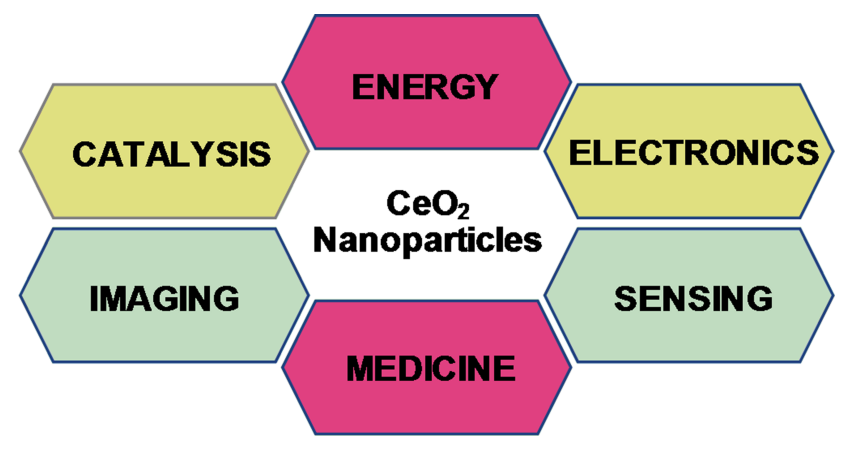

Figure I General applications of $\mathrm{CeO}_{2}$ nanoparticles.

such asketones, amines, enzymes, and phenols, which are believed to be responsible for the reduction and stabilization of bulk salts into respective nanoparticles NPs. ${ }^{16,19}$

To date various applications of green synthesized $\mathrm{CeO}_{2}$ NPs have been reported such as antimicrobial, anticancer, anti-larvicidal, photo-catalysis, and antioxidant therapies. ${ }^{16,20,22}$ Among other biomedical applications the antimicrobial potential is certainly the most exploited. Previously it has been reported that $\mathrm{CeO}_{2}$ NPs to display their anti-microbial actions through various mechanisms. ${ }^{9}$ But mostly $\mathrm{CeO}_{2}$ NPs kill microbes via triggering the production of an excess of reactive oxygen species in cells. ${ }^{9,16}$ However, further studies need to be conducted to fully elucidate the complete mechanism of action. Here in this review we aim to focus on the following topics. Arrays of biological resources have been exploited to date for the synthesis of $\mathrm{CeO}_{2}$ NPs. Moreover, the synthesis mechanisms along their biomedical applications are discussed with special emphasis on the antimicrobial activity.

\section{Synthesis of $\mathrm{CeO}_{2} \mathrm{NPs}$}

Nanoparticles are synthesized through various physico-chemical methods. ${ }^{5}$ However, both methods require toxic solvents, high temperature, and pressure, which pose threats to the environment. ${ }^{23,25}$ Moreover, higher cost, laborious downstream processing, lesser biocompatibility, instability, and low yield make them further inefficient. ${ }^{7,10}$ There is a growing need to fabricate nanostructures which have the potential to solve these problems. 5 .9 Presently, researchers have exploited the green method to overcome all these challenges. ${ }^{5}$ For instance, plants, microbes, and other biological products have been used as reducing and/ or stabilizing agents in the fabrication of ecofriendly $\mathrm{NPs}^{25} \mathrm{CeO}_{2}$ NPs have also been synthesized using various physical, chemical, and biological methods. ${ }^{9}$ The latter is extensively utilized for its biomedical, pharmacological, and food applications due to their safe and biocompatible nature. ${ }^{9}$ Moreover, features like high yield, everlasting stability, and better morphologies can be obtained using a greener approach. ${ }^{7,9}$

\section{Green Synthesis from Plants}

Green syntheses of $\mathrm{CeO}_{2}$ NPs have been reported using plant extracts, microbial, and other biological derivatives. Plants in this regard have been the most efficient source due to their abundance, safe nature, and rich source of reducing and stabilizing agents. ${ }^{26,29}$ Various parts of plants such as leaves, flower, and stem have been used for the synthesis of $\mathrm{CeO}_{2}$ NPs. ${ }^{16,30,31}$ Till date the majority of green synthesis studies have been conducted on leaves extracts, as it is a rich source of metabolites. ${ }^{11,16,32,33}$ A broad variety of metabolites/phytochemicals in plant extracts such as ketones, carboxylic acids, phenols, and ascorbic acid are used as reduction and stabilizing agents (Figure 2). Plants based $\mathrm{CeO}_{2}$ NPs are produced through a simple approach in which bulk metal salt is mixed with the extract and the reaction completes in minutes to a few hours in ordinary lab conditions. ${ }^{28,29,34}$ The metallic salt solution is reduced into respective nanoparticles via the phytochemicals whose synthesis is confirmed firstly through color change from colorless to yellowish, brownish, or whitish, and then characterized through various spectroscopic and imaging techniques. ${ }^{16,29,35}$

Leaf extract of Moringa oleifera $L$ was used to synthesize $\mathrm{CeO}_{2}$ NPs with spherical morphologies and size of $100 \mathrm{~nm}$. The synthesized NPs showed potential antimicrobial and wound healing properties. ${ }^{36}$ Gloriosa Superba leaf extract was used as a reducing and stabilizing agent in synthesis of $\mathrm{CeO}_{2}$ NPs and has shown potential antibacterial properties. ${ }^{37}$ Hibiscus sabdariffa natural extract yielded crystalline $\mathrm{CeO}_{2}$ with a diameter of $3.9 \mathrm{~nm}{ }^{30}$ Spherical shaped nanoparticles of size $63.6 \mathrm{~nm}$ synthesized from Gel extract of medicinally important plant Aloe Barbadensis. ${ }^{38}$ The resultant $\mathrm{CeO}_{2}$ NPs showed high antioxidant potential. Green synthesis of $\mathrm{CeO}_{2}$ nanoparticles was demonstrated using Jatropha curcus leaf extract having high photocatalytic activity and a monodispersed shape of 3-5 $\mathrm{nm} .{ }^{10}$ Spherical shaped cerium oxide nanoparticles are synthesized using Leaf extract of Oleo Europaea, with a size of $24 \mathrm{~nm}$ having high antimicrobial activity against both gram-negative and positive strains of bacteria. ${ }^{16}$ Origanum majorana extracts were used to synthesize $\mathrm{CeO}_{2} \mathrm{NPs}$, having pseudo 


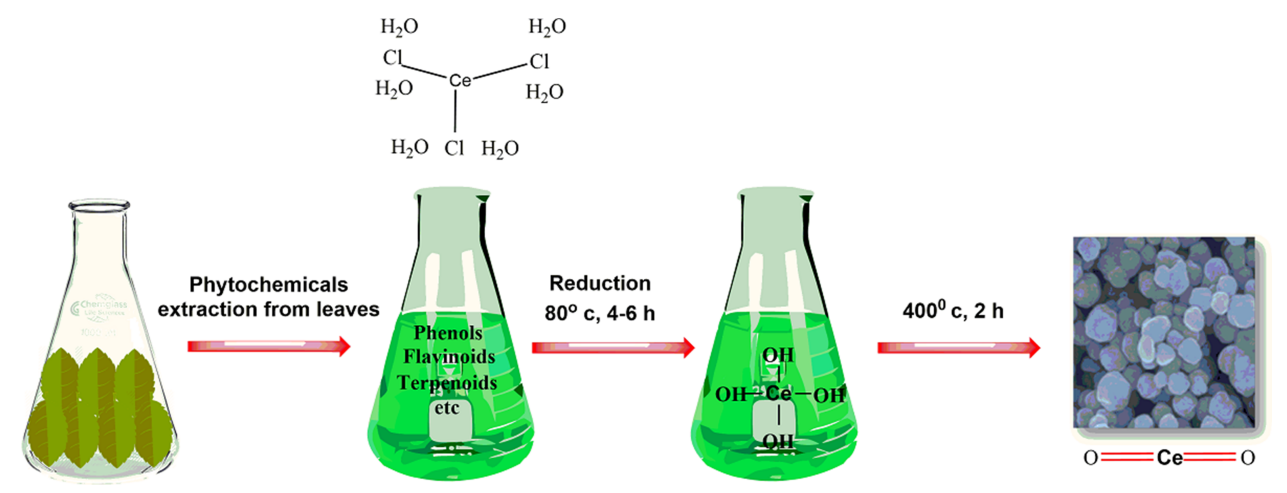

Figure 2 Biosynthesis: Reduction, stabilization and characterization of $\mathrm{CeO}_{2}$ nanoparticles.

spherical shape $(20 \mathrm{~nm})$. FT-IR confirmed that the reduction is attributed to the presence of different phenolic and flavonoids compounds in the extract. ${ }^{18} \mathrm{CeO}_{2}$ was synthesized using Rubia cordifolia leaf fusions. Spectroscopic and microscopic analysis revealed hexagonal shaped NPs having a size of $26 \mathrm{~nm}$. The biogenic $\mathrm{CeO}_{2} \mathrm{NPs}$ also showed excellent anti-cancer potential. ${ }^{33}$ Nano rod size ranges from 5-55 nm CeO ${ }_{2} \mathrm{NPs}$ resulted when Pedalium murex $\mathrm{L}$. was added to the aqueous solution of salt at room temperature having high antibacterial activity. ${ }^{32}$ China rose petal was used as a robust bio template for the facile fabrication of novel ceria Nano sheet with a size of about 7 $\mathrm{nm}^{39}$ The deviation in size and morphology noticed among the reported studies might be due to the different influence of reaction temperature, $\mathrm{pH}$, time, concentration of salt precursor or plants extracts, and part of the plant being used. ${ }^{13,27,28,40}$ Moreover, plants based $\mathrm{CeO}_{2}$ NP's showed excellent stability at diverse conditions. For instance, green mediated ceria NP's remain stable at liquid solution and no physiochemical changes were observed. ${ }^{13,36}$ Similarly, biogenic $\mathrm{CeO}_{2}$ NP's also showed high thermal stability at high temperature and remained stable for a longer period of time, which indicates their long durability and everlasting stability. ${ }^{27,28,33,41}$ Until now, various plants have been used in the biogenic synthesis of $\mathrm{CeO}_{2}$ NPs and are shown in Table 1.

\section{Green Synthesis from Microbes}

Microbes also have an intrinsic potential to synthesize nanoparticles, as they are a rich source of secondary metabolites. ${ }^{23}$ Among other nanoparticles $\mathrm{CeO}_{2}$ NPs with various shapes and sizes have been synthesized in recent years from microbes (Table 2). Green synthesis of $\mathrm{CeO}_{2}$ from microbial species is a simple, reliable, cost-effective, and eco-friendly approach. ${ }^{42}$ Microbial metabolites such as enzymes, proteins, and heterocyclic derivatives play a crucial role in reducing and stabilizing of $\mathrm{CeO}_{2}$ bulk salt into respective NPs. ${ }^{42,43}$ Moreover, micro-biogenic $\mathrm{CeO}_{2} \mathrm{NPs}$ exhibited improved stability, water dispensability, and showed high fluorescent properties and were less agglomerated. ${ }^{43}$

Aspergillus niger extract yielded cubic fluorite NPs with spherical morphology and an average size of $5 \mathrm{~nm}$. FT-IR analysis revealed the presence of an hydroxyl group, carboxylic group, and phenol group which are supposed to be involved in the reduction of NPs. ${ }^{21}$ Curvularia lunata extract has also been used to synthesize spherical shaped $\mathrm{CeO}_{2}$ NPs with size ranges from 5-20 $\mathrm{nm}$. Color change from white to yellow brown indicated initial reaction. The NPs were tested against microbial pathogens and showed excellent antibacterial potential. Spherical shaped $\mathrm{CeO}_{2}$ NPs of size ranges from 20-30 $\mathrm{nm}$ were made using Fusarium solani extract which showed effective growth inhibition and biofilm formation of pathogenic bacterial strains. ${ }^{42}$ Shadab Ali Khan observed the biosynthesis of spherical shaped (12-20 nm) $\mathrm{CeO}_{2}$ NPs by using a thermophilic fungus Humicola capping agent. ${ }^{43}$ The resultant NPs were characterized by UV, XPS, PL- Spectroscopy, TEM, FT-IR, and XRD. Moreover, these NPs showed excellent potential in treatment of neurodegenerative disorders such as Alzheimer's and Parkinson's diseases. Bacterial extract has also been exploited in the fabrication of $\mathrm{CeO}_{2} \mathrm{NPs}$, for instance Bacillus subtilis extract yielded spherical shaped NPs with an average size of $8 \mathrm{~nm}$. The bacterial mediated NPs also showed excellent antioxidant potential in vitro. ${ }^{44}$ Despite, all these applications the microbial route of synthesis has certain shortcomings, such as the high probability of pathogenicity, laborious culturing, and contamination issues. However, it offers a lot of promise in the field of nanotechnology and could become a leading avenue in 
Table I $\mathrm{CeO}_{2}$ Nanoparticles Made from Various Plants Species

\begin{tabular}{|c|c|c|c|c|c|c|c|}
\hline $\begin{array}{l}\text { S. } \\
\text { No }\end{array}$ & Plant Name & Part & Characterization & Shape & $\begin{array}{l}\text { Size } \\
(\mathrm{nm})\end{array}$ & FT-IR Group & Ref \\
\hline I. & $\begin{array}{l}\text { Gloriosa } \\
\text { superba }\end{array}$ & Leaf & XRD, XPS, TEM, FT-IR \& UV- vis & Spherical & 5 & $\begin{array}{l}\text { O-H, Ce-OH, H-O-H \& } \\
\text { O-C-O }\end{array}$ & [37] \\
\hline 2. & $\begin{array}{l}\text { Hibiscus } \\
\text { sabdariffa }\end{array}$ & Flower & HRTEM, SEM, XRD, XPS, EDS, FT-IR & & 3.9 & $\mathrm{Ce}-\mathrm{OH}$ & [30] \\
\hline 3. & Olive & Leaf & SEM, XRD, FT-IR, TGA & Spherical & 6 & $\mathrm{C}-\mathrm{O}, \mathrm{O}-\mathrm{H} \& \mathrm{H}-\mathrm{H}$ & [26] \\
\hline 4. & Oleo Europaea & Leaf & XRD, SEM, TEM, UV- Vis, FT-IR, TGA & Spherical & 24 & $\mathrm{O}-\mathrm{H}, \mathrm{C}-\mathrm{H} \& \mathrm{~N}-\mathrm{O}$ & [16] \\
\hline 5. & $\begin{array}{l}\text { Prosopis } \\
\text { jujiflora }\end{array}$ & Leaf & $\begin{array}{l}\text { UV-vis, PSA, FT-IR, XRD, XPS \& } \\
\text { HRTEMRTE }\end{array}$ & Spherical & 15 & $\mathrm{O}-\mathrm{H} \& \mathrm{Ce}-\mathrm{O}$ & [II \\
\hline 6. & $\begin{array}{l}\text { Salvia } \\
\text { macrosiphon } \\
\text { Boiss }\end{array}$ & Seed & XRD, UV-Vis, FT-IR, FESEM \& TGA & Spherical & $\sim 47$ & $\mathrm{Ce}-\mathrm{O}, \mathrm{O}-\mathrm{Ce}-\mathrm{O}, \mathrm{C}-\mathrm{H} \& \mathrm{C}=\mathrm{O}$ & [22] \\
\hline 7. & Acalypha indica & Leaf & XRD, SEM, TEM, EDS \& FT-IR & Spherical & $25-30$ & $\mathrm{O}-\mathrm{H}, \mathrm{Ce}-\mathrm{OH} \& \mathrm{Ce}-\mathrm{O}-\mathrm{Ce}$ & [13] \\
\hline 8. & $\begin{array}{l}\text { Aloe } \\
\text { barbadensis }\end{array}$ & Leaf & XRD, TEM, FT-IR \& PSA & Spherical & 63.6 & $\begin{array}{l}-\mathrm{C}-\mathrm{H}-, \mathrm{C}=\mathrm{O},-\mathrm{C}-\mathrm{H}-, \mathrm{C}-\mathrm{F}, \\
=\mathrm{C}-\mathrm{H} \& \mathrm{C}-\mathrm{Cl}\end{array}$ & [38] \\
\hline 9. & Aloe vera & Leaf & FT-IR, XPS, HR-TEM & Spherical & $2-3$ & $-\mathrm{C}-\mathrm{H},-\mathrm{C}-\mathrm{C}$ & [19] \\
\hline 10. & Rubia cordifolia & Leaf & UV- Vis, XRD, XPS, SEM, FT-IR, EDAX & Hexagonal & 26 & $\mathrm{O}-\mathrm{H} \& \mathrm{Ce}-\mathrm{O}$ & [33] \\
\hline 11. & Prosopis farcta & Aerial & UV-Vis, PXRD, TEM, FESEM, FT-IR & Spherical & 30 & $\mathrm{O}-\mathrm{H}, \mathrm{Ce}-\mathrm{O}$ and $\mathrm{N}=\mathrm{O}$ & [58] \\
\hline 12. & China rose & Petal & FESEM, FETEM, AFM, XRD \& XPS & Nano-sheet & 7 & _ & [39] \\
\hline 13. & $\begin{array}{l}\text { Centella } \\
\text { asiatica }\end{array}$ & - & UV-vis, DLS, SEM, HRTEM \& EDS & Spherical & 8 & - & [59] \\
\hline 14. & Walnut & Shell & XRD, SEM, TEM, EDS \& FT-IR & Spherical & $9-12$ & $\mathrm{Ce}-\mathrm{O}$ and $\mathrm{O}-\mathrm{H}$ & [60] \\
\hline 15. & $\begin{array}{l}\text { Azadirachta } \\
\text { Indica }\end{array}$ & Leaf & $\begin{array}{l}\text { UV-Vis, XRD, XPS, FESEM, TEM, HRTEM, } \\
\text { EDS, UV-DRS \& FT-IR }\end{array}$ & Spherical & $10-15$ & $\mathrm{Ce}-\mathrm{O}-\mathrm{C}, \mathrm{O}-\mathrm{H}, \mathrm{C}-\mathrm{H}$ & [6I] \\
\hline 16. & $\begin{array}{l}\text { Euphorbia } \\
\text { tirucalli }\end{array}$ & Stem & PXRD, SEM, TEM, FT-IR \& PL & Flaky & $37-40$ & $-\mathrm{OH},-\mathrm{COO}-$ and $\mathrm{C}-\mathrm{H}$ & [28] \\
\hline 17. & Lemon grass & Grass & XRD, PL, TEM \& SAED & - & $10-40$ & - & [62] \\
\hline 18. & Leucas aspera & Leaf & PXRD, SEM, UV-vis, TEM, SAED & Microsphere & $4-13$ & - & [63] \\
\hline 19. & $\begin{array}{l}\text { Petroselinum } \\
\text { crispum }\end{array}$ & & UV-vis, IR-, XRD, SEM, TEM & Spherical & $50-15$ & - & [14] \\
\hline 20. & $\begin{array}{l}\text { Moringa } \\
\text { oleifera }\end{array}$ & Peel & UV- Vis, FT-IR, XRD \& HRTEM & Spherical & 45 & $\mathrm{Ce}-\mathrm{O} \& \mathrm{Ce}-\mathrm{O}-\mathrm{Ce}$ & [36] \\
\hline 21. & Watermelon & $\begin{array}{l}\text { Fruit } \\
\text { juice }\end{array}$ & PXRD, FT-IR, UV-Vis \& SEM & Irregular & 36 & $\begin{array}{l}\mathrm{Ce}-\mathrm{O}, \mathrm{C}=\mathrm{O}, \mathrm{C}-\mathrm{O}, \mathrm{C}-\mathrm{H} \& \\
\mathrm{O}-\mathrm{H}\end{array}$ & [64] \\
\hline 22. & Carrageenan & - & $\begin{array}{l}\text { PXRD, FT-IR, FESEM, UV-Vis, and TGA/ } \\
\text { DTA }\end{array}$ & Spherical & 34 & $-\mathrm{SO}_{3-}^{-}, \mathrm{Ce}-\mathrm{O}, \mathrm{H}-\mathrm{O}-\mathrm{H} \& \mathrm{C}-\mathrm{O}$ & [27] \\
\hline
\end{tabular}

(Continued) 
Table I (Continued).

\begin{tabular}{|c|c|c|c|c|c|c|c|}
\hline $\begin{array}{l}\text { S. } \\
\text { No }\end{array}$ & Plant Name & Part & Characterization & Shape & $\begin{array}{l}\text { Size } \\
(\mathrm{nm})\end{array}$ & FT-IR Group & Ref \\
\hline 23. & $\begin{array}{l}\text { Ceratonia } \\
\text { siliqua }\end{array}$ & Leaf & XRD, FESEM, TEM \& FT-IR & Spherical & 22 & - & [57] \\
\hline 24. & $\begin{array}{l}\text { Stevia } \\
\text { rebaudiana }\end{array}$ & - & XRD, FESEM, EDS, VSM & Spherical & $8-10$ & - & [65] \\
\hline 25. & $\begin{array}{l}\text { Salvadora } \\
\text { persica }\end{array}$ & & PXRD, FT-IR, UV-Vis, TEM, FESEM, EDS & Spherical & $10-15$ & $\begin{array}{l}\text { O-H, C-H, C=C, Ce-O \& } \\
\mathrm{C}-\mathrm{O} C\end{array}$ & [20] \\
\hline 26. & Morus nigra & Fruit & TEM, XRD \& UV-Vis & Irregular & $\sim 7.5$ & _ & [35] \\
\hline 27. & $\begin{array}{l}\text { Annona } \\
\text { muricata }\end{array}$ & Fruit & XRD, FT-IR, UV-DRS, FESEM & - & _ & - & [50] \\
\hline 28. & $\begin{array}{l}\text { Justicia } \\
\text { adhatoda }\end{array}$ & Leaf & XRD, SEM, TEM, FT-IR \& UV-DRS & Stick-like & $20-25$ & $\begin{array}{l}-\mathrm{OH}, \mathrm{C}-\mathrm{H}, \mathrm{C}=\mathrm{O}, \mathrm{N}=\mathrm{O}, \mathrm{C}=\mathrm{C}, \\
\mathrm{C}-\mathrm{O} \& \mathrm{C}-\mathrm{N}-\mathrm{C}\end{array}$ & [17] \\
\hline 29. & Jatropha curcus & Leaf & XRD, TEM \& UV-Vis & Monodispersed & $3-5$ & _ & [10] \\
\hline 30. & $\begin{array}{l}\text { Origanum } \\
\text { majorana }\end{array}$ & Leaf & TEM, FESEM, XRD \& FT-IR & Spherical & 20 & $\mathrm{O}-\mathrm{H}, \mathrm{C}-\mathrm{H} \& \mathrm{C}=\mathrm{O}$ & [18] \\
\hline 31. & $\begin{array}{l}\text { Pedalium } \\
\text { murex }\end{array}$ & Leaf & XRD, FT-IR, UV-Vis DRS, SEM & Nano-rod & $5-55$ & $\begin{array}{l}\mathrm{O}-\mathrm{H}, \mathrm{C}-\mathrm{H}, \mathrm{C}-\mathrm{O}, \mathrm{C}=\mathrm{O}, \mathrm{C}=\mathrm{N}, \\
\mathrm{C}-\mathrm{N} \& \mathrm{Ce}-\mathrm{O}\end{array}$ & [32] \\
\hline 32. & $\begin{array}{l}\text { Elaeagnus } \\
\text { angustifolia }\end{array}$ & Leaf & XRD, SEM, TEM, FT-IR & Spherical & $30-75$ & $\mathrm{C}-\mathrm{H} \& \mathrm{C}=\mathrm{O}$ & [29] \\
\hline 33. & $\begin{array}{l}\text { Euphorbia } \\
\text { amygdaloides }\end{array}$ & & TEM, SEM, XRD \& UV-vis & Spherical & 42 & - & [3I] \\
\hline 34. & Orange & Peel & XRD, TEM, FT-IR \& UV-vis & $\begin{array}{l}\text { Cubic } \\
\text { structure }\end{array}$ & $20-25$ & $\mathrm{C}-\mathrm{O}-\mathrm{H}$ or $\mathrm{C}-\mathrm{O}-\mathrm{R}$ & [34] \\
\hline 35. & Piper betle & Leaf & XRD, FT-IR, SEM, EDS, XPS \& TEM & - & - & $\mathrm{N}-\mathrm{H}, \mathrm{N}-\mathrm{O} \& \mathrm{C}-\mathrm{N}$ & [66] \\
\hline
\end{tabular}

nanomedicine, but is yet to be explored. Particularly, these accessories. Moreover, these biogenic NPs can also be microbial based NPs can be used in designing novel ferti- exploited in disease management, drug synthesis, and lizers, fabricating sterile surfaces, polymers, and medical delivery.

Table $2 \mathrm{CeO}_{2}$ Nanoparticles Synthesized from Various Fungus Species

\begin{tabular}{|c|c|c|c|c|c|c|}
\hline $\begin{array}{l}\text { S. } \\
\text { No }\end{array}$ & $\begin{array}{l}\text { Microbe } \\
\text { Name }\end{array}$ & Characterization & Shape & $\begin{array}{l}\text { Size } \\
\mathrm{nm}\end{array}$ & FT-IR Group & Ref \\
\hline I. & $\begin{array}{l}\text { Curvularia } \\
\text { lunata }\end{array}$ & TG/DTA, XRD, Raman, PL, FT-IR, UV-vis and TEM & Spherical & $5-20$ & - & [54] \\
\hline 3. & Humicola sp. & UV-Vis, XPS, PLS, TEM, FT-IR \& XRD & Spherical & $12-20$ & $\mathrm{Ce}-\mathrm{O} \& \mathrm{Ce}-\mathrm{O}-\mathrm{Ce}$ & [43] \\
\hline 4. & Fusarium solani & $\begin{array}{l}\text { FT-IR, PL, TG/DTA, FESEM, XRD, EDAX, TEM, XPS, SAED \& } \\
\text { CLSM }\end{array}$ & Spherical & $20-30$ & $-\mathrm{OH}, \mathrm{C}-\mathrm{N} \&-\mathrm{C}-\mathrm{O}-\mathrm{C}$ & [42] \\
\hline 5. & Aspergillus niger & UV-Vis, FT-IR, XPS, XRD, TG/DTA, PL \& TEM & Spherical & $5-20$ & $\begin{array}{l}\mathrm{H}-\mathrm{O}-\mathrm{H}, \mathrm{O}-\mathrm{C}-\mathrm{O} \& \mathrm{Ce}- \\
\mathrm{O}\end{array}$ & {$[21]$} \\
\hline
\end{tabular}




\section{Green Synthesis from Biological Products}

Apart from the synthesis of nanomaterial from eukaryotes and prokaryotes organisms, NPs have also synthesized from biological derivatives. ${ }^{23}$ They also play a crucial role in the reduction and stabilization of NPs. ${ }^{23,25}$ In contrast to plants and the microbial approach, bio-product based $\mathrm{CeO}_{2}$ are much safer, scalable, and have shown excellent biocompatibility. ${ }^{45,47}$ For instance, egg white protein was used in order to synthesize $\mathrm{CeO}_{2}$ NPs having size ranges from 8-17 nm with spherical morphologies. ${ }^{48}$ These NPs were characterized and confirmed by UV's, FT-IR, TGA/DTA, and PXRD. FT-IR analysis revealed that the phenol, ether, hydroxyl, and amide groups were responsible for the reduction of these NPs. It also showed a good in vitro cytotoxicity effect towards human periodontal fibroblasts cells. Agarose is a natural matrix and has been used as a stabilizing and capping agent for $\mathrm{CeO}_{2}$ NPs. NPs obtained have spherical morphologies with a diameter of $10.5 \mathrm{~nm}$. NPs were characterized using various methods, including UV, FESEM, FT-IR, TGA/DTA, PXRD, and TGA/DTA techniques. As revealed by FT-IR analysis, it was found that the hydroxyl, ether, phenol, and amide groups were involved in biosynthesis. ${ }^{45}$ Starch has also been exploited as a novel source for the synthesis of nano-ceria, with the results revealing spherical shape NPs with a diameter of $6 \mathrm{~nm} .^{12}$
Spherical shaped $\mathrm{CeO}_{2} \mathrm{NPs}$, with a size of 5-10 nm, were synthesized from dextran. The resultant NPs exhibited strong anti-cancer potential. ${ }^{46}$ Gum tragacanth reported by Darroudi et $\mathrm{al}^{49}$ was used in the biosynthesis of $\mathrm{CeO}_{2} \mathrm{NPs}$. These NPs were mono-dispersed shape with an average size range from 20-40 $\mathrm{nm}$. The $\mathrm{CeO}_{2}-\mathrm{NPs}$ exhibited very low cytotoxic effects on Neuro2A cell lines, making them suitable candidates for various biomedical and pharmacological applications. ${ }^{49}$ Some other biological products which have been used for synthesis of $\mathrm{CeO}_{2}$ NPs are listed in Table 3 . Despite their biological applications these biogenic NPs could be used as a promising candidate in diseases treatment, drug delivery, and packing food. Some other products have also been explored for the synthesis of $\mathrm{CeO}_{2} \mathrm{NPs}$ which are shown in Table 3.

\section{Biological Activity of Green-Mediated Cerium Oxide Nanoparticles}

Antimicrobial Activity of Green-Mediated Cerium Oxide Nanoparticles

In the last few years, nanotechnology-based therapies have been exploited in disease diagnostics, treatment, and

Table 3 Bio-Mediated $\mathrm{CeO}_{2}$ Nanoparticles from Different Sources of Biological Products

\begin{tabular}{|c|c|c|c|c|c|c|}
\hline $\begin{array}{l}\text { S. } \\
\text { No }\end{array}$ & Name & Characterization & Shape & $\begin{array}{l}\text { Size } \\
\mathrm{nm}\end{array}$ & FT-IR Group & Ref \\
\hline $\mathrm{I}$. & Egg protein & UV-Vis, FESEM, FT-IR, TGA/DTA \& PXRD & Spherical & $8-17$ & $\begin{array}{l}-\mathrm{OH}, \mathrm{Ce}-\mathrm{O}, \mathrm{Ce}-\mathrm{O}-\mathrm{Ce}, \\
\mathrm{C}-\mathrm{H} \& \\
\mathrm{~N}=\mathrm{O}\end{array}$ & [48] \\
\hline 2. & Honey & UV-Vis, FESEM, FT-IR, TGA/DTA, EDS \& PXRD & Spherical & 23 & $\begin{array}{l}\mathrm{O}-\mathrm{H}, \mathrm{Ce}-\mathrm{O}, \\
\mathrm{Ce}-\mathrm{O}-\mathrm{Ce}, \mathrm{Ce}-\mathrm{O}-\mathrm{C} \& \\
\mathrm{~N}=\mathrm{O}\end{array}$ & [67] \\
\hline 3. & Agarose & UV-Vis, FESEM, FT-IR, TGA/DTA \& PXRD & Spherical & 10.5 & $\begin{array}{l}\mathrm{O}-\mathrm{H}, \mathrm{Ce}-\mathrm{O}, \mathrm{Ce}-\mathrm{O}-\mathrm{Ce} \& \\
\mathrm{~N}=\mathrm{O}\end{array}$ & [45] \\
\hline 4. & Starch & UV-Vis, PXRD \& TEM & Spherical & 6 & _ & [12] \\
\hline 5. & Dextran & TEM, DLS, XPS \& UV-Vis & Spherical & $5-10$ & - & [46] \\
\hline 6. & $\begin{array}{l}\text { Polyethylene } \\
\text { glycol }\end{array}$ & HRTEM, TEM, UV-Vis \& SLS-DLS & Spherical & $\sim 2$ & - & [68] \\
\hline 7. & Chitosan & XRD, HRTEM, FT-IR, TGA/DTA \& UV-Vis & Spherical & $\sim 4$ & - & [69] \\
\hline 8. & Pectin & $\begin{array}{l}\text { DLS, FESEM, EDSS, XRD, FT-IR, NMR, PL, FESEM, } \\
\text { EDSS \& UV-Vis }\end{array}$ & Spherical & $\leq 40$ & $\begin{array}{l}-\mathrm{OH}, \mathrm{C}-\mathrm{H}, \mathrm{C}=\mathrm{O}, \\
-\mathrm{O}-\mathrm{CH} 3, \mathrm{C}-\mathrm{O}-\mathrm{C}, \mathrm{N}=\mathrm{O} \& \\
\mathrm{Ce}-\mathrm{O}\end{array}$ & [47] \\
\hline 9. & Tannic acid & FT-IR, XPS, XRD, HRTEM \& UV-visible & Polycrystalline & $\sim 10$ & - & {$[15]$} \\
\hline
\end{tabular}




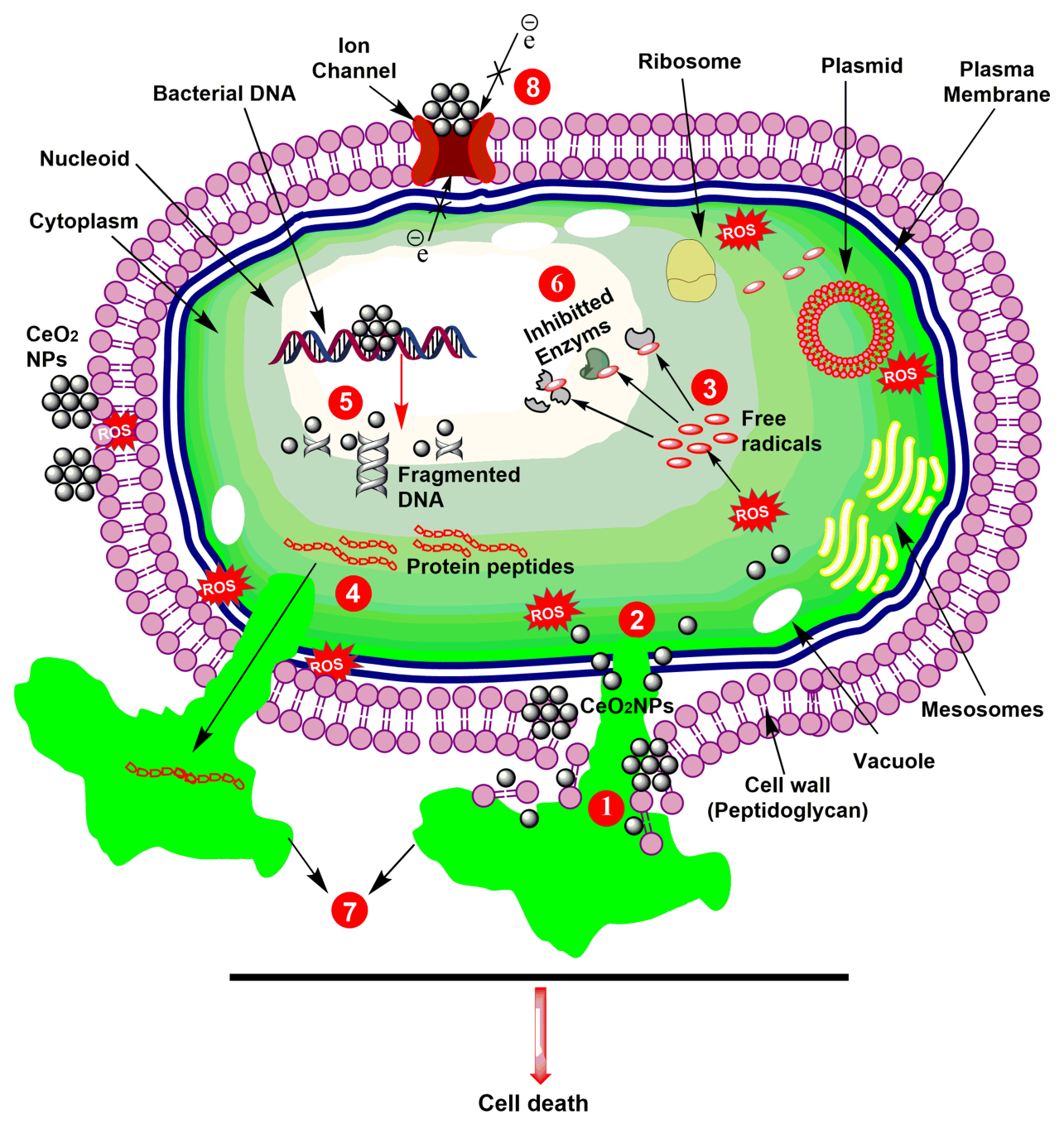

Figure 3 Schematic representation of antibacterial activity of $\mathrm{CeO}_{2}$ nanoparticles. (I) Cell wall disruption; (2) Cell membrane disintegration; (3) Free radicals productions; (4) Loss of protein peptides; (5) DNA fragmentation; (6) Vital enzymes inhibition; (7) Loss of cellular fluids; and (8) Disruption in electron transport.

formulations of novel drugs. ${ }^{1}$ For instance, the antimicrobial potential of NPs has been mostly exploited; and has showed substantial outcomes. ${ }^{23,24}$ Presently, $\mathrm{CeO}_{2}$ NPs have attracted great interest as an antimicrobial agent, in particular against bacterial pathogens. ${ }^{15,16,50}$ The exact mechanism of killing microbes is yet not clearly elucidated. However, it is proposed that $\mathrm{CeO}_{2} \mathrm{NPs}$ mostly kill microbes via a massive production of reactive oxygen species (ROS) in cells, as shown in Figure 3. ${ }^{15,51}$ The bactericidal potential of $\mathrm{CeO}_{2} \mathrm{NPs}$ is attributed to strong electrostatic properties, distinctive morphologies, small size, and low band energy. ${ }^{16,52}$ Due to strong electrostatic potential $\mathrm{CeO}_{2} \mathrm{NPs}$ interact with membrane proteins thiols groups, which results in protein denaturation, membrane impermeability eventually leads to microbial death ${ }^{42,53}$ (Figure 3). Exposure to $\mathrm{CeO}_{2}$ NPs kills microbes via membrane collapse by attachment with mesosomes, malfunctioning of cellular compartments, and bio organic molecules, which ultimately lead to abnormal metabolism and physiology. ${ }^{16,42}$ Similarly, green mediated NPs killpathogens in a similar fashion and various biological species have been exploited and tested against a wide variety of microbes ${ }^{13,16}$ (Table 4). However, biogenic $\mathrm{CeO}_{2} \mathrm{NPs}$ unique morphologies, small size and bio-compatible nature were found to be more effcient and have the potential to treat a wide range of pathogenic bacterial species. ${ }^{11,13,15,16,42}$ Moreover, it also has the potential to kill both gram-positive and gram-negative bacteria, but due to structural complexity of gram-negative bacteria's membranes, it is more sensitive against gram-positive species. ${ }^{13,21,37,47,54}$ The difference in antimicrobial activity is due to differences in electrostatics between NPs and the bacterial wall, plant species, and wall composition. 
Table 4 Various Microbes Tested Against Biogenic $\mathrm{CeO}_{2}$ Nanoparticles

\begin{tabular}{|c|c|c|c|}
\hline $\begin{array}{l}\text { S. } \\
\text { NO }\end{array}$ & Source & Microbes Tested & Ref \\
\hline I. & Olea europaea & $\begin{array}{l}\text { Fungus (Aspergillus flavus, Fusarium solani, and Aspergillus niger) } \\
\text { Bacterial sps (Staphylococcus aureus, Escherichia coli, Pseudomonas aeruginosa and Klebsiella pneumonia) }\end{array}$ & {$[26]$} \\
\hline 2. & Moringa oleifera & S. aureus and $E$. coli & {$[36]$} \\
\hline 3. & Curvularia lunata & $\begin{array}{l}\text { Staphylococcus aureus, Streptococcus pneumoniae and Bacillus subtilis, Pseudomonas aeruginosa, Proteus vulgaris and } \\
\text { Klebsiella pneumoniae }\end{array}$ & {$[54]$} \\
\hline 4. & Leucas aspera & Klebsiella aerogenes, Escherichia coli, Pseudomonas desmolyticum and Staphylococcus aureus & {$[63]$} \\
\hline 5. & Acalypha indica & Escherichia coli and Staphylococcus aureus & {$[13]$} \\
\hline 6. & Annona muricata & Enterococcusfaecalis, Staphylococcus aureus, Klebsiella pneumonia and Escherichia coli & {$[50]$} \\
\hline 7. & Gloriosa Superba & $\begin{array}{l}\text { Staphylococcus aureus and Streptococcus pneumonia, E.coli, Proteus vulgaris, Klebsiella pneumonia, Shigella dysenteriae } \\
\text { and Pseudomonas aeruginosa }\end{array}$ & {$[37]$} \\
\hline 8. & Aspergillus niger & Streptococcus pneumoniae, Bacillus subtilis, Proteus vulgaris and Escherichia coli & {$[21]$} \\
\hline 9. & Fusarium solani & Staphylococcus aureus, Pseudomonas aeruginosa, Escherichia coli and Klebsiella pneumonia & {$[42]$} \\
\hline 10. & Justicia adhatoda & Staphylococcus aureus and Escherichia coli & {$[17]$} \\
\hline II & $\begin{array}{l}\text { Euphorbia } \\
\text { amygdaloides }\end{array}$ & P. acidilactici & {$[3 \mid]$} \\
\hline 12 & Pectin & E. coli and B. subtilis & {$[47]$} \\
\hline
\end{tabular}

Due to rapid evolution of the bacterial genome, bacteria have evolved to confer resistance to antimicrobial agents. ${ }^{55,56}$ Thus, in quest of a new treatment, biogenic $\mathrm{CeO}_{2} \mathrm{NPs}$ has shown promising results in treating multidrug resistance bacteria and could be a promising candidate against such refractory pathogenesis. ${ }^{26} \mathrm{CeO}_{2}$ NPs along other conjugates have been amalgamated with various organic and inorganic hybrids to enhance the antimicrobial response. ${ }^{17,46}$ Similarly, bio-mediated $\mathrm{CeO}_{2}$ NPs kills fungi by producing a mass number of free radicals and ROS which causes distorted structure and physiology and leads to fungus death. ${ }^{16}$ However, a few studies have only been conducted on fungi. Despite the increasing knowledge on the antimicrobial activity of $\mathrm{CeO}_{2} \mathrm{NPs}$ much remains unknown about their exact mechanism of encountering bacteria, toxicity, in vivo studies, and environmental concerns, which needs to be addressed. Moreover, the low band energy potential of $\mathrm{CeO}_{2}$ NPs could be used in fabricating sterile surfaces at hospital or lab settings and will diminish nosocomial and other acquired infections.

\section{Other Potential Biomedical Applications}

Beside anti-microbial therapies $\mathrm{CeO}_{2} \mathrm{NPs}$ have also been used in management of other ailments. ${ }^{35,57}$ For instance, biogenic $\mathrm{CeO}_{2} \mathrm{NPs}$ have been mostly used in treatment of various cancers, such as osteosarcoma, colon, cervical, and breast cancers. ${ }^{18,20,27,46,52}$ Results indicated that these NPs exhibited strong anticancer potential and could be used as a chemotherapeutic agent thanks to their minimal toxicity, capacity to induce apoptosis, and/or necrosis in cancer cells. ${ }^{46} \mathrm{CeO}_{2}$ NPs synthesized from Origanum majorana and Ceratonia siliqua showed high antioxidant activity. ${ }^{18,31,57}$ Results showed higher expression of antioxidant enzymes which in turn eradicated free radicals and improved cellular functions. ${ }^{31}$ Furthermore, antioxidant potential was higher when compared to commercial synthetic antioxidants. ${ }^{18} \mathrm{CeO}_{2}$ NPs synthesized from fruit extract of Morus nigra exhibited excellent anti-diabetic activity on L6 cell lines. The treatment was dosage dependent and NPs with lesser size resulted in higher uptake of glucose in vitro. ${ }^{35}$ Though biogenic $\mathrm{CeO}_{2}$ NPs have shown excellent pharmacological potential, however, the mechanism of action, minimum inhibitory concentration, and best possible delivery system should need to be determined. Moreover, cytotoxicity and genotoxicity should be tested in vivo models to evaluate the compatibility in both in vivo and in vitro models. 


\section{Conclusions and Future Prospects}

In this paper we have reviewed the recent trends and understandings of biogenic $\mathrm{CeO}_{2}$ NPs and their pharmacological applications. Various sources such as plants, microbes, and other biological products have been discussed with the mechanism of synthesis and their biomedical applications. Due to their unique surface morphologies, crystal small nature, and biocompatible nature biogenic $\mathrm{CeO}_{2}$ NPs have got phenomenal interest in biomedical and other fields. For instance, it has been used in treating various cancers, antimicrobial, and antioxidant therapies. In particular, the green synthesized nanoparticles have shown significant antimicrobial potential against a wide range of bacterial species. The mechanism of combating such pathogens have also been elucidated and supposed to be due to the mass production of reactive oxygen species and deactivation of scavenging enzymes. The ROS impedes the membranes, disrupts the cellular compartments, and disintegrates the bio organic molecules and hampers the associated functions and ultimately causes death. It has also shown promising results against multi-drug bacteria, and could be a potential antimicrobial agent in future against such refractory pathogens. However, further studies should conduct in vivo models, to reveal the full mechanism alongside any side-effects. Moreover, it has also shown excellent anticancer and antioxidant potential in vitro setups, but their toxicity and dosage are yet unknown, which needs to be addressed. Despite their role in various therapies their mechanism of synthesis needs to be optimized, whereas in vivo evaluation as well as toxicity should be further screened.

\section{Abbreviations}

$\mathrm{CeO}_{2} \mathrm{NPs}$, cerium oxide nanoparticles; DGA, differential thermal analysis; DSL, dynamic light scattering; EDS, energy-dispersive X-ray spectroscopy; FESEM, field emission scanning electron microscopy; FT-IR, Fourier transform infrared spectroscopy; HRTEM, high-resolution transmission electron microscopy; PL, photoluminescence; PXRD, powder X-ray diffraction; ROS, reactive oxygen species; SLS, static light scattering; TEM, transmission electron microscopy; TGA, thermal gravimetric analysis; UV-Vis, UV-visible spectroscopy; XPS X-ray, photoelectron spectrometry; XRD, X-ray diffraction.

\section{Disclosure}

The authors report no conflicts of interest for this work.

\section{References}

1. Kubik T, Bogunia-Kubik K, Sugisaka M. Nanotechnology on duty in medical applications. Curr Pharm Biotechnol. 2005;6(1):17-33. doi:10.2174/1389201053167248

2. Bhushan B. Springer Handbook of Nanotechnology. Springer; 2017.

3. Jianrong $C$, Yuqing $M$, Nongyue $H$, et al. Nanotechnology and biosensors. Biotechnol Adv. 2004;22(7):505-518. doi:10.1016/j. biotechadv.2004.03.004

4. Smith DM, Simon JK, Baker Jr JR Jr. Applications of nanotechnology for immunology. Nat Rev Immunol. 2013;13(8):592-605. doi:10.1038/nri3488

5. Mohanraj V, Chen Y. Nanoparticles-a review. Trop J Pharm Res. 2006;5(1):561-573.

6. Das S, Dowding JM, Klump KE, et al. Cerium oxide nanoparticles: applications and prospects in nanomedicine. Nanomedicine. 2013;8 (9):1483-1508. doi:10.2217/nnm.13.133

7. He L, Su Y, Lanhong J, et al. Recent advances of cerium oxide nanoparticles in synthesis, luminescence and biomedical studies: a review. J Rare Earths. 2015;33(8):791-799. doi:10.1016/S10020721(14)60486-5

8. Walkey C, Das S, Seal S, et al. Catalytic properties and biomedical applications of cerium oxide nanoparticles. Environ Sci. 2015;2 (1):33-53. doi:10.1039/C4EN00138A

9. Rajeshkumar S, Naik P. Synthesis and biomedical applications of cerium oxide nanoparticles-a review. Biotechnol Rep. 2018;17:1-5. doi:10.1016/j.btre.2017.11.008

10. Magudieshwaran R, Ishii J, Raja KCN, et al. Green and chemical synthesized $\mathrm{CeO} 2$ nanoparticles for photocatalytic indoor air pollutant degradation. Mater Lett. 2019;239:40-44. doi:10.1016/j.matlet. 2018.11.172

11. Arunachalam T, Karpagasundaram M, Rajarathinam N. Ultrasound assisted green synthesis of cerium oxide nanoparticles using Prosopis juliflora leaf extract and their structural, optical and antibacterial properties. Mater Sci Poland. 2017;35(4):791.

12. Darroudi M, Sarani M, Kazemi Oskuee R, et al. Green synthesis and evaluation of metabolic activity of starch mediated nanoceria. Ceram Int. 2014;40(1):2041-2045. doi:10.1016/j.ceramint.2013.07.116

13. Kannan S, Sundrarajan M. A green approach for the synthesis of a cerium oxide nanoparticle: characterization and antibacterial activity. Int $J$ Nanosci. 2014;13(03):1450018. doi:10.1142/ S0219581X14500185

14. Korotkova A, Borisovna PO, Aleksandrovna GI, et al. "Green" synthesis of cerium oxide particles in water extracts petroselinum crispum. Curr Nanomater. 2019;4(3):176-190. doi:10.2174/ 2405461504666190911155421

15. Kumar KM, Mahendhiran M, Diaz MC, et al. Green synthesis of Ce3 + rich $\mathrm{CeO} 2$ nanoparticles and its antimicrobial studies. Mater Lett. 2018;214:15-19. doi:10.1016/j.matlet.2017.11.097

16. Maqbool Q, Nazar M, Naz S, et al. Antimicrobial potential of green synthesized $\mathrm{CeO}(2)$ nanoparticles from Olea europaea leaf extract $<1$ sub>. Int J Nanomedicine. 2016;11:5015-5025. doi:10.2147/IJN. S113508

17. Nithya P, Murugesan B, Sonamuthu J, Samayanan S, Mahalingam S. Facile biological synthetic strategy to morphologically aligned $\mathrm{CeO}$ 2/ZrO 2 core nanoparticles using Justicia adhatoda extract and ionic liquid: enhancement of its bio-medical properties. $J$ Photochem Photobiol B. 2017;178.

18. Nezhad S, Haghi A, Homayouni M. Green synthesis of cerium oxide nanoparticle using Origanum majorana L. leaf extract, its characterization and biological activities: green synthesis of nanoparticle. Appl Organomet Chem. 2019;34:e5314.

19. Dutta D, Mukherjee R, Patra M, et al. Green synthesized cerium oxide nanoparticle: a prospective drug against oxidative harm. Colloids Surf B Biointerfaces. 2016;147:45-53. doi:10.1016/j. colsurfb.2016.07.045 
20. Miri A, Darroudi M, Sarani M. Biosynthesis of cerium oxide nanoparticles and its cytotoxicity survey against colon cancer cell line. Appl Organomet Chem. 2019.

21. Gopinath K, Karthika V, Sundaravadivelan C, et al. Mycogenesis of cerium oxide nanoparticles using Aspergillus niger culture filtrate and their applications for antibacterial and larvicidal activities. J Nanostructure Chem. 2015;5(3):295-303. doi:10.1007/s40097-0150161-2

22. Elahi B, Mirzaee M, Darroudi M, et al. Preparation of cerium oxide nanoparticles in salvia macrosiphon boiss seeds extract and investigation of their photo-catalytic activities. Ceram Int. 2018;45 (4):4790-4797. doi:10.1016/j.ceramint.2018.11.173

23. Nadeem M, Tungmunnithum D, Hano C, et al. The current trends in the green syntheses of titanium oxide nanoparticles and their applications. Green Chem Lett Rev. 2018;11(4):492-502. doi:10. 1080/17518253.2018.1538430

24. Nadeem M, Abbasi BH, Younas M, et al. A review of the green syntheses and anti-microbial applications of gold nanoparticles. Green Chem Lett Rev. 2017;10(4):216-227. doi:10.1080/1751825 3.2017.1349192

25. Srikar SK, Giri DD, Pal DB, et al. Green synthesis of silver nanoparticles: a review. Green Sustain Chem. 2016;6(01):34. doi:10.4236/ gsc.2016.61004

26. Maqbool Q, Nazar M, Maqbool A, et al. $\mathrm{CuO}$ and $\mathrm{CeO} 2$ nanostructures green synthesized using olive leaf extract inhibits the growth of highly virulent multidrug resistant bacteria. Front Pharmacol. 2018;9 (987). doi:10.3389/fphar.2018.00987.

27. Nourmohammadi E, Kazemi Oskuee R, Hasanzadeh L, et al. Cytotoxic activity of greener synthesis of cerium oxide nanoparticles using carrageenan towards a WEHI 164 cancer cell line. Ceram Int. 2018;44(16):19570-19575. doi:10.1016/j.ceramint.2018.07.201

28. Malleshappa J, Nagabhushana H, Prashantha SC, et al. Eco-friendly green synthesis, structural and photoluminescent studies of $\mathrm{CeO} 2$ : eu3+nanophosphors using E. tirucalli plant latex. J Alloys Compd. 2014;612:425-434. doi:10.1016/j.jallcom.2014.05.101

29. Singh A, Hussain I, Singh NB, et al. Uptake, translocation and impact of green synthesized nanoceria on growth and antioxidant enzymes activity of Solanum lycopersicum L. Ecotoxicol Environ Saf. 2019;182:109410. doi:10.1016/j.ecoenv.2019.109410

30. Thovhogi N, Diallo A, Gurib-Fakim A, et al. Nanoparticles green synthesis by Hibiscus sabdariffa flower extract: main physical properties. $J$ Alloys Compd. 2015;647:392-396. doi:10.1016/j. jallcom.2015.06.076

31. Nadaroglu H, Onem H, Alayli Gungor A. Green synthesis of Ce2O3 NPs and determination of its antioxidant activity. IET Nanobiotechnol. 2017;11(4):411-419.

32. Nithya P, Murugesan B, Sonamuthu J, Samayanan S, Mahalingam S. [BMIM] PF6 ionic liquid mediated green synthesis of ceramic $\mathrm{SrO} /$ $\mathrm{CeO} 2$ nanostructure using Pedalium murex leaf extract and their antioxidant and antibacterial activities. Ceram Int. 2019.

33. Sisubalan N, Ramkumar VS, Pugazhendhi A, et al. ROS-mediated cytotoxic activity of $\mathrm{ZnO}$ and $\mathrm{CeO} 2$ nanoparticles synthesized using the Rubia cordifolia L. leaf extract on MG-63 human osteosarcoma cell lines. Environ Sci Pollut Res. 2018;25(11):10482-10492. doi:10.1007/s11356-017-0003-5

34. Sultan Irshad M, Aziz MH, Fatima M et al. Green synthesis, cytotoxicity, antioxidant and photocatalytic activity of $\mathrm{CeO} 2$ nanoparticles mediated via orange peel extract (OPE). Mater Res Express. 2019.

35. Rajan A, Rajan A, John A, Philip D. Green synthesis of $\mathrm{CeO} 2$ nanostructures by using Morus nigra fruit extract and its antidiabetic activity. AIP Conf Proc. 2019;2105:020008.

36. Tammineni S, Roopan S. Photocatalytic and antibacterial properties of phytosynthesized of $\mathrm{CeO} 2$ NPs using Moringa oleifera peel extract. J Photochem Photobiol B. 2016;161.
37. Arumugam A, Karthikeyan C, Hameed AS et al. Synthesis of cerium oxide nanoparticles using Gloriosa superba L. leaf extract and their structural, optical and antibacterial properties. Mater Sci Eng C. 2015;49.

38. Priya G, Kanneganti A, Kumar KA, Rao KV, Bykkam S. Bio synthesis of cerium oxide nanoparticles using aloe barbadensis miller gel. Int J Sci Res Publ. 2014;4.

39. Qian J, Chen F, Zhao X, et al. China rose petal as biotemplate to produce two-dimensional ceria nanosheets. J Nanopart Res. 2011;13 (12):7149-7158. doi:10.1007/s11051-011-0626-2

40. Nadaroglu H, Onem H, Gungor AA. Green synthesis of Ce2O3 NPs and determination of its antioxidant activity. IET Nanobiotechnol. 2016;11(4):411-419. doi:10.1049/iet-nbt.2016.0138

41. Zamani A, Marjani AP, Alimoradlu K. Walnut shell-templated ceria nanoparticles: green synthesis, characterization and catalytic application. Int J Nanosci. 2018;17(06):1850008.

42. Kunga Sugumaran V, Gopinath K, Palani NS et al. Plant pathogenic fungus F. solani mediated biosynthesis of Nanoceria: antibacterial and antibiofilm activity. RSC Adv. 2016;6:42720-9.

43. Khan SA, Ahmad A. Fungus mediated synthesis of biomedically important cerium oxide nanoparticles. Mater Res Bull. 2013;48 (10):4134-4138. doi:10.1016/j.materresbull.2013.06.038

44. Pitchumani Krishnaveni M, Annadurai G. Biosynthesis of nanoceria from bacillus subtilis: characterization and antioxidant potential. Res J Life Sci. 2019;5(3):644.

45. Kargar H, Ghasemi F, Darroudi M. Bioorganic polymer-based synthesis of cerium oxide nanoparticles and their cell viability assays. Ceram Int. 2015;41(1):1589-1594. doi:10.1016/j.ceramint.2014.09.095

46. Alpaslan E, Yazici H, Golshan NH, et al. pH-dependent activity of dextran-coated cerium oxide nanoparticles on prohibiting osteosarcoma cell proliferation. ACS Biomater Sci Eng. 2015;1 (11):1096-1103. doi:10.1021/acsbiomaterials.5b00194

47. Patil SN, Paradeshi JS, Chaudhari PB, et al. Bio-therapeutic potential and cytotoxicity assessment of pectin-mediated synthesized nanostructured cerium oxide. Appl Biochem Biotechnol. 2016;180 (4):638-654. doi:10.1007/s12010-016-2121-9

48. Kargar H, Ghazavi H, Darroudi M. Size-controlled and bio-directed synthesis of ceria nanopowders and their in vitro cytotoxicity effects. Ceram Int. 2015;41(3, Part A):4123-4128. doi:10.1016/j. ceramint.2014.11.108

49. Darroudi M, Sarani M, Kazemi Oskuee R, et al. Nanoceria: gum mediated synthesis and in vitro viability assay. Ceram Int. 2014;40 (2):2863-2868. doi:10.1016/j.ceramint.2013.10.026

50. Sebastiammal S, Mariappan A, Neyvasagam K, Fathima AL. Annona muricatainspired synthesis of $\mathrm{CeO} 2$ nanoparticles and their antimicrobial activity. Mater Today. 2019;9:627-632.

51. Xia T, Kovochich M, Liong M, et al. Comparison of the mechanism of toxicity of zinc oxide and cerium oxide nanoparticles based on dissolution and oxidative stress properties. ACS Nano. 2008;2 (10):2121-2134. doi: $10.1021 / \mathrm{nn} 800511 \mathrm{k}$

52. Abbas F, Iqbal J, Jan T, et al. Differential cytotoxicity of ferromagnetic $\mathrm{Co}$ doped $\mathrm{CeO} 2$ nanoparticles against human neuroblastoma cancer cells. J Alloys Compd. 2015;648:1060-1066. doi:10.1016/j. jallcom.2015.06.195

53. Tong G-X, Du -F-F, Liang Y, et al. Polymorphous $\mathrm{ZnO}$ complex architectures: selective synthesis, mechanism, surface area and Zn-polar plane-codetermining antibacterial activity. J Mater Chem B. 2013;1(4):454-463. doi:10.1039/C2TB00132B

54. Munusamy S, Bhakyaraj K, Vijayalakshmi L, Stephen A, Narayanan V Synthesis and characterization of cerium oxide nanoparticles using Curvularia lunata and their antibacterial properties. Int J Innov Res Sci Eng. 2014;2(1):318.

55. Davies J, Wright GD. Bacterial resistance to aminoglycoside antibiotics. Trends Microbiol. 1997;5(6):234-240. doi:10.1016/ S0966-842X(97)01033-0 
56. Livermore DM. Bacterial resistance: origins, epidemiology, and impact. Clin Infect Dis. 2003;36(Supplement_1):S11-S23. doi:10.10 $86 / 344654$

57. Javadi F, Taghavizadeh Yazdi ME, Baghani M, et al. Biosynthesis, characterization of cerium oxide nanoparticles using Ceratonia siliqua and evaluation of antioxidant and cytotoxicity activities. Mater Res Express. 2019;6(6):065408. doi:10.1088/2053-1591/ab08ff

58. Miri A, Sarani M. Biosynthesis, characterization and cytotoxic activity of $\mathrm{CeO} 2$ nanoparticles. Ceram Int. 2018;44(11):12642-12647. doi:10.1016/j.ceramint.2018.04.063

59. Sankar V, SalinRaj P, Athira R, et al. Cerium nanoparticles synthesized using aqueous extract of Centella asiatica: characterization, determination of free radical scavenging activity and evaluation of efficacy against cardiomyoblast hypertrophy. $R S C A d v$. 2015;5 (27):21074-21083. doi:10.1039/C4RA16893C

60. Zamani A, Marjani AP, Alimoradlu K. Walnut shell-templated ceria nanoparticles: green synthesis, characterization and catalytic application. Int J Nanosci. 2017;17(06):1850008. doi:10.1142/ S0219581X18500084

61. Sharma JK, Srivastava P, Ameen S, et al. Phytoconstituents assisted green synthesis of cerium oxide nanoparticles for thermal decomposition and dye remediation. Mater Res Bull. 2017;91:98-107. doi:10.1016/j.materresbull.2017.03.034

62. Maensiri S, Labuayai S, Laokul P, et al. Structure and optical properties of $\mathrm{CeO} 2$ nanoparticles prepared by using lemongrass plant extract solution. Jpn J Appl Phys. 2014;53(6S):06JG14. doi:10. 7567/JJAP.53.06JG14
63. Malleshappa J, Nagabhushana H, Sharma SC, et al. Leucas aspera mediated multifunctional $\mathrm{CeO} 2$ nanoparticles: structural, photoluminescent, photocatalytic and antibacterial properties. Spectrochim Acta A Mol Biomol Spectrosc. 2015;149:452-462. doi:10.1016/j.saa.2015.04.073

64. Reddy Yadav LS, Manjunath K, Archana B, et al. Fruit juice extract mediated synthesis of $\mathrm{CeO} 2$ nanoparticles for antibacterial and photocatalytic activities. Eur Phys J Plus. 2016;131(5):154. doi:10.1140/ epjp/i2016-16154-y

65. Khatami M, Sarani M, Mosazadeh F, et al. Nickel-doped cerium oxide nanoparticles: green synthesis using stevia and protective effect against harmful ultraviolet rays. Molecules. 2019;24(24):4424. doi:10.3390/molecules24244424

66. Pinheiro D, Sunaja Devi KR, Jose A, et al. Experimental design for optimization of 4-nitrophenol reduction by green synthesized $\mathrm{CeO} 2$ / g-C3N4/Ag catalyst using response surface methodology. $J$ Rare Earths. 2019. doi:10.1016/j.jre.2019.10.001

67. Darroudi M, Hoseini SJ, Kazemi Oskuee R, et al. Food-directed synthesis of cerium oxide nanoparticles and their neurotoxicity effects. Ceram Int. 2014;40(5):7425-7430. doi:10.1016/j. ceramint.2013.12.089

68. Qi L, Fresnais J, Muller P, et al. Interfacial activity of phosphonated-PEG functionalized cerium oxide nanoparticles. Langmuir. 2012;28(31):11448-11456. doi:10.1021/la302173g

69. Kaushik A, Solanki PR, Pandey MK, et al. Cerium oxide-chitosan based nanobiocomposite for food borne mycotoxin detection. Appl Phys Lett. 2009;95(17):173703. doi:10.1063/1.3249586
International Journal of Nanomedicine

\section{Publish your work in this journal}

The International Journal of Nanomedicine is an international, peerreviewed journal focusing on the application of nanotechnology in diagnostics, therapeutics, and drug delivery systems throughout the biomedical field. This journal is indexed on PubMed Central, MedLine, CAS, SciSearch ${ }^{\mathbb{R}}$, Current Contents ${ }^{\mathbb{B}} /$ Clinical Medicine, $^{2}$
Journal Citation Reports/Science Edition, EMBase, Scopus and the Elsevier Bibliographic databases. The manuscript management system is completely online and includes a very quick and fair peer-review system, which is all easy to use. Visit http://www.dovepress.com/ testimonials.php to read real quotes from published authors. 